\title{
ABSTRACTS FOR MILLISECOND PULSAR TIMING SESSION
}

1) Arecibo: millisecond pulsar timing results

Victoria M. Kaspi and Joseph Taylor

Joseph Henry Laboratories and Physics Department, Princeton University

Long-term, High-Precision Timing of Millisecond Pulsars

Biweekly timing observations of PSRs B1937+21 and B1855+09 have been made at the 305-m radio telescope at Arecibo Observatory in Puerto Rico for 9 and 8 years respectively. At each observing epoch we measure pulse arrival times for PSR B1855+09 at $1.4 \mathrm{GHz}$ with typical uncertainties of 1 microsecond, and arrival times for PSR $\mathrm{B} 1937+21$ at 1.4 and $2.3 \mathrm{GHz}$ with uncertainties of 0.2 microsecond. The pulse arrival times are fitted to a simple model for each pulsar, yielding high-precision astrometric, rotational, and orbital parameters, and a diverse range of conclusions on topics including astrometry, cosmology, and interstellar medium physics. In the residual pulse arrival times, particularly for PSR B1937+21, we have observed small systematic trends not explained by our deterministic model. We discuss possible origins of these trends, and show that the most likely explanation is phenomena intrinsic to the pulsar itself.

\section{2) Arecibo Gregorian Upgrade Program}

Donald B. Campbell and Michael M. Davis

A major upgrading of the Arecibo $305 \mathrm{~m}$ diameter radio telescope is currently underway consisting of: 1) a $16 \mathrm{~m}$ high wire mesh screen around the perimeter of the telescope's primary reflector, which will reduce system noise temperatures by shielding the focal area from thermal emission from the surrounding terrain; 2) replacing the 'line feeds' currently used to correct for the spherical aberration inherent in the telescope's design with a Gregorian reflector system; 3) increasing the power of the 2,380 $\mathrm{MHz}$ transmitter used for planetary studies to $1.0 \mathrm{Mwatts}$. These changes will result in increased sensitivity at all frequencies above $1 \mathrm{GHz}$, large instantaneous bandwidths and increased frequency coverage from the current maximum of 2 to $3 \mathrm{GHz}$ to about $8 \mathrm{Ghz}$. Table 1 , gives a detailed comparison of the expected performance of the new system compared with the current one. 
Table 1:

\begin{tabular}{|l|l|l|}
\hline & Present Performance & Expected Performance \\
\hline Effective Aperture & $22.000 \mathrm{~m}^{2}$ & $30.000 \mathrm{~m}^{2}$ \\
\hline Maximum Sensitivity & $8 \mathrm{~K} / \mathrm{Jy}$ & $11 \mathrm{~K} / \mathrm{Jy}$ \\
\hline Best System Temperature & $35 \mathrm{~K}$ & $20 \mathrm{~K}$ \\
\hline Transmitter Power & $0.42 \mathrm{MW}$ & $1.0 \mathrm{MW}$ \\
\hline Maximum Frequency & $2.4 \mathrm{GHz}$ & $8 \mathrm{GHz}$ \\
\hline Instantaneous Bandwidth & $40 \mathrm{MHz}$ & $\geq 500 \mathrm{MHz}$ \\
\hline Minimum Beamwidth & 130 arcseconds & 40 arcseconds \\
\hline Pointing Accuracy & 15 arcseconds & 5 arcseconds \\
\hline
\end{tabular}

3) Timing Array Results from Green Bank Donald C. Backer

Pulsars with millisecond rotation periods have been demonstrated to be highly stable $-10^{-14}$ or less for durations of one year. Observations of pulse arrival times allow us to link Earth atomic clocks to these celestial clocks. A spatial array of millisecond pulsars can be used for a number of fundamental investigations. Pulsar timing array experiments are under way at a number of radio observatories around the globe.

Our group began pulsar timing array observations in 1987 with two pulsars - 1821-24 and $1937+21$ - at the NRAO $42 \mathrm{~m}$ telescope in Green Bank, WV. Data is obtained every two months at 800 and $1,400 \mathrm{MHz}$ using a $2 \times 20 \mathrm{MHz}$ spectral processor. This summer a new, coherent dispersion removal processor was brought on line. We also began weekly observations with a $25 \mathrm{~m}$ telescope of two objects at $610 \mathrm{MHz}$.

Results for $1821-24$ and $1937+21$ display similar instability on long time scales as has been reported from Nancay and Arecibo, respectively. Current interpretations of millisecond pulsar instability is that the stars themselves are undergoing erratic rearrangment of their internal structure driven by spin down.

4) BIPM analysis of millisecond pulsar data

G. Petit

\section{Bureau International des Poids et Mesures}

Millisecond pulsars are galactic objects that exhibit a very stable spining period, so that their rotational phase may be used as a time reference, named pulsar time. Although it is not possible this way to define a time scale (Guinot and Petit, 1991), pulsar time may be compared to atomic time to get some insight on the long term stability of atomic time. Several tens of millisecond pulsars have now been discovered, which opens the possibility that an average time scale may be deduced through a long-term 
stability algorithm. Such an ensemble average makes it possible to reduce the level of the instabilities originating from the pulsars or from other sources of noise, which are unknown but independent.

We have presented the basis for such an algorithm (Petit et al., 1992) and applied it to real pulsar data. It is shown that pulsar time could shortly become more stable than the present atomic time, for averaging times of a few years. Pulsar time can also be used as a flywheel to maintain the accuracy of atomic time in case of temporary failure of the primary standards, or to transfer the improved accuracy of future standards back to the present.

Another interesting aspect is that pulsars in a binary system can be used to completely define a time scale through their orbiting phase. Such a binary pulsar time, with a potential accuracy in the 10-12 region, would be the best astronomical realization of a time scale.

In collaboration with the CNES, french space agency, we have developed a technique to determine the time of arrival of the pulsar signals at a radiotelescope (Petit and Fayard, 1994). Such a technique could help to obtain more pulsar data, a necessary step for the computation of an ensemble pulsar time.

Guinot, B., Petit, G., "Atomic time and the rotation of pulsars". Astron. Astrophys., 248,292-296, 1991.

Petit, G., Thomas, C., Tavella, P., "An ensemble pulsar time", Proc. 24th PITI meeting. 73-85, 1992.

Petit, G., Fayard, T., "First millisecond pulsar timing at seven observatories". 1994 (in submission).

5) A Method of Reducing the Variance in Millisecond Pulsar Timing

Alex Lepek, Fred L. Walls and David W. Allan

The variance $\sigma^{2}(\tau)$ is defined as $\left\langle\left(\chi_{i}-2 \chi_{i+\tau}+\chi_{i+2 \tau}\right)^{2}\right\rangle=\langle\delta \delta\rangle$; where $\delta$ is a generalized time series. We define $\# \sigma^{2}(\tau)$ as $<\delta_{a} \delta_{b}>$, where $\delta_{a}$ is the generalized time series obtained with one measurement system and $\delta_{b}$ from a different system.

If one clock is common then we show that in the presence of noise, the limit as $\mathrm{n}$ goes to infinity of $\# \sigma^{2}(\tau)$ approaches the common clock. $\sigma_{i}(\tau)$. This is not the case when computing the $\mathrm{N}$-cornered-hat $\sigma_{b}(\tau)$ for the common clock in the presence of noise.

We propose to use the \# $\sigma$ method to obtain a better estimate of the timing $\sigma(\tau)$ of a pulsar which is considered here as the common clock and the other two clocks are signals from two pulsar radio receivers. Conversely, the common clock could be an internationally connected reference clock from the best weighted set of international clocks optimally combined together. The net of our analysis is that with the outstanding international set of atomic clocks and with several observatories gathering data on 
some 30 millisecond pulsar timing signals, whose long-term stability is of great interest, efficiency in data analysis will become increasingly important.

6) Instabilities in TAI

Nichola Koshelayevski, VNIIFTRI

Frequency steps of the order of $1 \times 10^{-14}$ have been observed. Since these are of the same order as the instabilities in some of the millisecond pulsar data, this creates a metrology problem. These steps are outside of the occasional steering to bring the SI second into agreement with the primary standards.

Since contributing clocks appear to have better stability than TAI at some times, perhaps a study of how to use these high performing clocks would be useful. The goal should be to best utilize the international time keeping resources so that the reference time scales can be as good as reliable as possible.

\section{QUESTIONS, COMMENTS AND ANSWERS DURING THE SESSION:}

B. Guinot - question of V. Kaspi

In the graph showing time residuals for PSR $1937+21$, is the contribution of the second order derivative of period removed? Kaspi responded that the period derivative and other lower-order fitting parameters had been removed.

Alak Ray - question of V. Kaspi

What is the currently available level of accuracy with which pulsar timing can be done in some of the globular clusters (i.e. magnitude of the residuals for example in M15)? No answer recorded.

David Shaffer - question of V. Kaspi (\& Alan)

How do you relate time at Arecibo to TT(BIPM)? Response: via GPS used in common-view mode.

Simon Johnston - question of D. Backer

At high frequencies (about $10 \mathrm{GHz}$ ), pulse profiles are generally narrower and the effects of the ISM are greatly reduced. Can you comment on future high frequency observations with the new GBT? Backer responded that this is one of the motivations for going to the higher frequencies. Better understanding of the ISM as well as of millisecond pulsar characteristics and in general better metrology should result.

V. Kaspi - question of M. Davis

When can we expect high frequency observations again? Response: August 1995. 
D. Backer - question of A. Lepek

Can you clarify the application of this technique to a pulsar observation? I don't see that such a measurement relative to clock A or clock B has independent noise terms. Response: When one pulsar, which is the common clock, is measured by two independent receivers the uncommon measurement noisee may be reduced.

B. Guinot - coment in response to Koshelayevski's presentation

It is known that TAI suffers from an annual perturbation due to environmental effects on clocks. This effect is progressively reduced by use of better clocks and by the input of clocks in the Southern hemisphere, but it still remains a small residual annual tern. I would strongly advise to use TT(BIPM) for pulsar studies, because the annual term is removed by reference to primary standards of PTB.

N. Koshelayevski - comment in response to Guinot's

I think your point of TAI being less stable than TA(lab) is well understood. Steering a time scale can make it less stable.

M. Davis - question

How soon can pulsar data begin to contribute to international time-keeping? G. Guinot responded, "Ten years or more."

\section{SYNTHESIS OF ACTIVITY IN MILLISECOND PULSAR TIMING}

In order to achieve good millisecond pulsar timing, the following items are needed:

1) One or more millisecond pulsars with good spin-rate stability - including adequate modeling of the period derivative;

2) Pulsars with good signal to noise;

3) An adequate number of pulsars so that emsembling can be used;

4) An adequate period of time so that their is sufficient data on several pulsars in order to utilize them as an ensemble;

5) A reference terrestrial time scale with good long-term stability so that it can be used to help measure the stability of each of the contributing pulsars;

6) Adequate time transfer accuracy between the observing sites so as to not degrade the stability measurements of the pulsars against the reference clock;

7) Adequate models and theories for the solar system dynamics, the pulsar dynamos, the relativity and for the delays through the interstellar medium and earth's atmosphere;

8) Optimum acquisition and processing of the available dat; and

9) Provide as good a test bed as possible for the detection of gravitational waves; in this regard, one requirement is to have the pulsars nominally orthogonally distributed 
across the sky.

Since the IAU and as part of the reports given in The Hague 1994 Millisecond Pulsar Timing Session, significant progress has been reeported in all of the above nine items. In what follows, we will enumerate some of the progress.

1) Since the first millisecond pulsar (PSR 1937+21) was discovered in October 1982 , Backer reported at our session more than 30 pulsars now being measured. The spinrate stability on PSR $1855+09$ has been shown to be better than $1 \times 10^{\mathbf{1 4}}$. There is apparently still a need in how to best model the period derivative and the period second-derivative. Currently, there is not uniformity in approach.

2) PSR $1937+21$ has had a signal to noise as measured from Arecibo such that the measured noise shows white-noise time residuals at a level of about 300 nanoseconds. One of the most recent pulsars has even stronger signal as measured at Parks in Australia. A new dispersion removal processor has been installed at Green Bank, and with a new $100 \mathrm{~m}$ telescope measurement noise residuals with current pulsars may reach 100 ns. A new upgrade at Arecibo should be finished by summer of 1996 - giving it a larger surface area, a lower operating noise-temperature and more efficient focal system, which should be able to operate up to $8 \mathrm{GHz}$. It is expected to have measurement residuals at more than one site perhaps less than $100 \mathrm{~ns}$ within about two years.

3) The number of millisecond pulsars is about 30 with adequate timing stability to be useful for timing purposes. We are only now bginning to gather data on these; so we are only at the beginning of using these in some ensemble sense. The current integration time is of the order of several months to years to average down the measurement time residual in order to see either the pulsar stability or to have the stability be comparable to Earth reference clocks. In a general sense, this means we are only getting about one time-data point per year per pulsar per site. A decade's worth of data will be extremely helpful.

4) Because the signal strength is different from each pulsar and because the receiver signal to noise is different for each site, it will be expedient to combine and coordinate the data usage from the sites. There are now eleven sites taking data. The BIPM would be the logical coordination agency, if this could be agreen upon among the observers for timing purposes.

5) After the outstanding long-term stability of millisecond pulsars was documented - specifically that of PSR $1937+21$ - Dr. B. Guinot used the clocks contributing to TAI and UTC to construct ä after-the-fact very stable terrestrial reference time scale. This scale was denoted Terrestrial Time for a particular year; e.g. TT (BIPM -90). Since annual variations have been observed TAI and UTC during the 1980s, these could be removeed in thee TT scales. It has provided and excellent reference for the timing of millisecond pulsars.

Significant progress has also been made in TAI and UTC. At the beginning of the 
1990 s, the long-term stability of hydrogen-maser frequency standards has been documented to be better than $1 \times 10^{-14}$ for integration times of the order of months. These are now contributing to TAI and UTC. During the last couple of years, a new commercial cesium-beam frequency standard becaame available - the Hewlett Packard model 5071A. This standard has built in digital-servo techniques which reduce the effects of the environment on the long-term stability of this type of standard by about an order of magnitude. The frequency stabilities for this model have been documented to be in the range from 2 to $5 \times 10^{-15}$. There are now about 50 of these models contributing to TAI and UTC of the total of about 200. Annual variations are no longer apparent and the stability of TAI and UTC is now better than $1 \times 10^{-14}$. New studiees are under way to take better advantage of these new kinds of clocks.

Much new work has been done in developing more accurate primary cesium-beam frequency standards for the determination of the SI second. These include the use of optical pumping for the energy-state selection and cooled-cesium fountains. The former has reported an accuracy of $1 \times 10^{-14}$ and the latter seem to have potential accuracies of $1 \times 10^{-15}$ or potentially $1 \times 10^{-16}$. An accuracy of $1 \times 10^{-14}$ has been reported. These standards can potentially contribute significantly to the long-term frequency stability of TAI and UTC as well as that of TT, which is, of course, what is desired for timing millisecond pulsars.

6) In 1984 a GPS common-view time transfer system was installed at Arecibo to improve its accuracy to better than $50 \mathrm{~ns}$. This improved the Arecibo timing significantly over the previous Loran-C technique. The GPS common-view technique has been adopted as the international best operative method for time transfer. It is used for almost all of the clocks contributing to TAI and UTC. If all the errors are accounted for using this technique, accuracies of about 3 or 4 ns have been documented.

What is important for timing millisecond pulsars is not time accuracy, but long-term frequency stability and that each pulsar measurement is traceable to the best terrestrial frequency reference. While GPS common-view provides this capability as well as providing time-transfer accuracy, it has the disadvantage that a data exchange between the two sites being compared has to take place. This is now much automated.

Because of the inconvenience of transferring data in the GPS common-view approach, alternative techniques have been studied. Because of the degradation of the GPS broadcast signal for military security reasons (called Selective-Availability (SA)), the raw broadcast time signal may have peak variations from UTC by 200 ns. A simple daily average of all satellites averages most of the SA, and yields a day-to-day time stability better than 10 ns. "This, of course, like the GPS common-view approach provides time after the fact. It also provides UTC (USNO MC) to within a guarantied $100 \mathrm{~ns}$; in fact, it is usually within $20 \mathrm{~ns}$. In turn UTC (USNO MC) is almost always within 100 ns of UTC.

A new approach, called Enhanced GPS, has been developed for the telecommunications industry, which can provide a real time estimate of the broadcast UTC (USNO MC) 
broadcast by GPS. This has been documented to have a day-to-day stability of about $1.5 \mathrm{~ns}$ when the right clocks are used. This is accomplished by an optimum filtering of the SA.

In the future, with time-scale algorithm. research being implemented and with the utilization of the currently available very stablee clocks, it has been shown that it is possible to have a time scale such as UTC(USNO MC) synchronized to UTC to better than 20 ns. If such were accomplished, this would provide the near ideal reference for a millisecond pulsar timing site. If an Enhanced GPS system were installed, it could be used to both $\log$ the data as a tie to the international reference with full appreciation of the SI second in the long-term.

7) The precise metrology tool millisecond pulsars have provided for studies in astrometry has been a major boon to this work. The large number of papers that have been written in this area of work since the first one was discovered in 1982 is sufficient evidence. Some examples include: the discovery of very small binary partners to some of the millisecond pulsars - in a recent case more than one - and being able to determine precise orbits and relative masses, the determination that the assumed fixed glactic coordinate system may be slightly rotating; detailed measurements of the total electron content along these paths often previously thought to be constant (it häs been found to behave more like a random-walk process); and high accuracy and full use of the general theory of relativity arising from gravitational effects, velocity effects and the effective mass of radio photons as influenced by solar system masses. The current relativity theory has sufficient accuracy for measurements at thee $1 \times 10^{-16}$ level.

8) Optimum acquisitions and processing of the availablee data is very important given the cost of acquiring the data and the large benefits to science that have accrued and surely will yet. As unprecedented precisions in metrology have been the focus for more than three decades with the time and frequency community, some benefits are expected as a marriage of the techniques developed here are incorporated in the timing of millisecond pulsars. Some independently organized mullisecond pulsar timing workshops have been organized during the last 10 years with significant exchanges occurring between the participants as well as good coordination of metrology efforts.

Some examples of relevant time and frequency techniques are the following. It has ben shown that if the timing residuals have a white spectrum, then the uncertainty on the frequency estimate is given by $2^{*} \operatorname{Mod} . \sigma_{y}(\tau)$, where $\tau$ is the averaging time. This function integrates as $\tau^{-3 / 2}$. For millisecond pulsar timing, $\tau$ can be replaced by $\tau_{o}^{*} \mathrm{n}$ where $\tau_{o}$ is the nominal data spacing and $\mathrm{n}$ is the number of independent data points. For Arecibo, for example, $\tau_{o}$ is about two weeks in the measurements of PSR 1937+21. Heretofore, each pulsar has been analyzed at each site. If the data for a particular pulsar could be combined as a weighted set from all of the current 11 observing sites, this could have major impact on the rapidity with which the frequency (or period) uncertainty could be deduced. Since the number of data points could accumulate from all the sites and since the uncertainty is proportional to $\mathrm{n}^{-3 / 2}$, this could help overcome one current big limitation in millisecond pulsar timing - pulsar signal to noise. 
The importance of this is illustrated in the accompanying Mod. $\sigma_{y}(\tau)$ diagram. Here we see that if we have a measurement noise of $100 \mathrm{~ns}$ and a measurement period of about two weeks, then it would take an integration time of 200 years to reach $1 \times 10^{-18}$ - an accuracy goal hoped to be achieved within a couple of decades by the primary frequency standards community. Proper processing of the data would help greatly to overcome this measurement noise problem. This area needs further study and should be very useful.

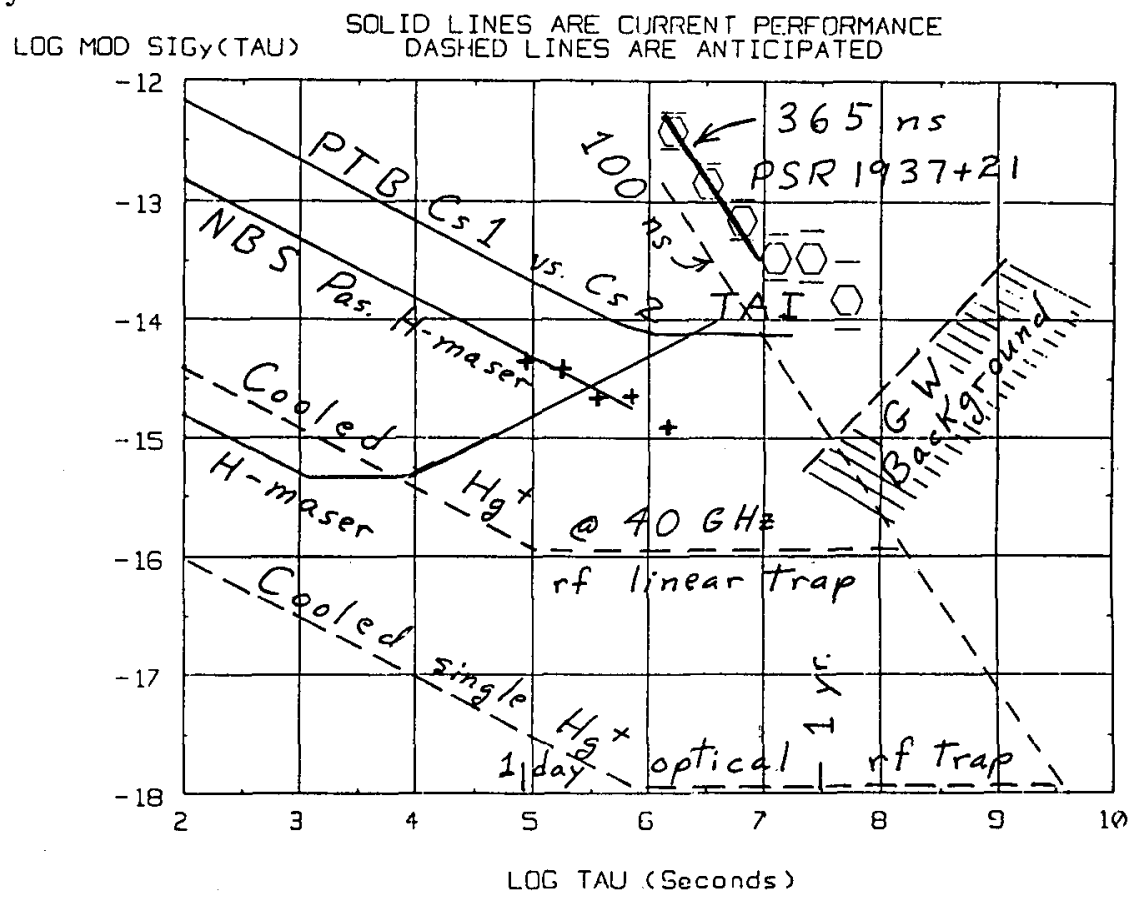

Relative to this proper use of data, another time and frequency metrology technique was introduced by Dr. Lepek at the 20 August special millisecond pulsar timing session. He has developed a \# $\sigma$, which is a way of averaging away the measurement noise so that one can truly observe the clock instabilities. This technique has been tested at NIST and other timing centers and works well. It will work with any second moment statistical measure - Whether in the frequency domain or in the time domain. One example off his technique would be to use two pulsars to investigate the stability of TT, for example, averaging away the measurement noise. He showed in some simulations that the measurement noise averages as $n^{-1 / 4}$, and that this technique is significantly superior to the three or $\mathrm{N}$-cornered hat approach for det'ermining an individual clock's instabilities.

Some studies have been done on efficient ways of estimating frequency (or period) drift in clocks in the presence of low-frequency noise. More work is also needed in this area. 